\title{
FUZZY CONTROL OF THE SPRAYING MEDICINE CONTROL SYSTEM
}

\author{
Yan Shi ${ }^{1}$, Chunmei Zhang ${ }^{2 *}$, Anbo Liang ${ }^{3}$, Haibo Yuan ${ }^{2}$ \\ ${ }^{1}$ Architecture and engineering college, Qingdao Agricultural University, Qingdao, China, \\ 266109 \\ ${ }^{2}$ College of Engine \& Electronic Engineering, Qingdao Agricultural University, Qingdao, \\ China., 266109 \\ ${ }^{3}$ International Intercommunion School, Qingdao Agricultural University, Qingdao, China, \\ 266109 \\ * Corresponding author, Address: College of Engine \& Electronic Engineering, Qingdao \\ Agricultural University, 700 Changcheng Road, Qingdao, 266109, P. R. China, \\ Tel: +13863901209, Email: zcm9092@126.com
}

Abstract: The continuous spraying was adopted by most of spraying pesticide systems, which can not accord to the extent and the characteristic change of targets to automatically regulate dose of drugs. This way not only resulted in waste of pesticides, but also caused pollution of environment. The designing studied the variable spraying pesticide system of real-time, which based sensory technology, at the same time, fuzzy control was applied in this system. The paper introduced the composition and control principle of the spraying pesticide system, at the other hand, the linking of Simulink and the fuzzy controller was introduced by this paper. Fuzziness of inputs and the outputs, the determination of subordinate functions, the establishment of fuzzy controlling rules and fuzzy control simulation of the spraying system were designed. Simulating result indicated that transiting time is short and the system is steady.

Keywords: fuzzy control, variable spraying pesticide, Matlab, Simulink

\section{INTRODUCTION}

Mechanization of Chinese plant protection is relatively laggard in China, the mechanization of plant protection about accurate spraying pesticide is

Shi, Y., Zhang, C., Liang, A. and Yuan, H., 2008, in IFIP International Federation for Information Processing, Volume 259; Computer and Computing Technologies in Agriculture, Vol. 2; Daoliang Li; (Boston: Springer), pp. 1087-1094. 
also the same. In recent years, our country intensified research in this area and has made a great deal of progress. For example, Zhao Maocheng and others in Nanjing Forestry University designed the variable spraying pesticide control system based on trees feature which can accurately spray pesticide to targets. He Xiongkui and others in China Agricultural University carried on the design of electrostatic spraying machine to targets about orchards and experiment study.

At present, study of variable spraying pesticide machines mainly has the map-based automatic spraying pesticide system to targets and the automatic spraying pesticide system to targets in real-time sensory technology. Performance of the former is not strong, but, the latter has the advantages in this area (Shi Yan, 2004).

This paper designed a variable spraying pesticide which could conduct decision of spraying pesticide according to weed area, the control system marches variable spraying according to decision of spraying pesticide.

\section{MATIERIALS AND METHOD}

This part introduced designing process of the control system.

\subsection{The total structure of spraying pesticide system}

Figure 1 is the total block diagram of the spraying pesticide system.

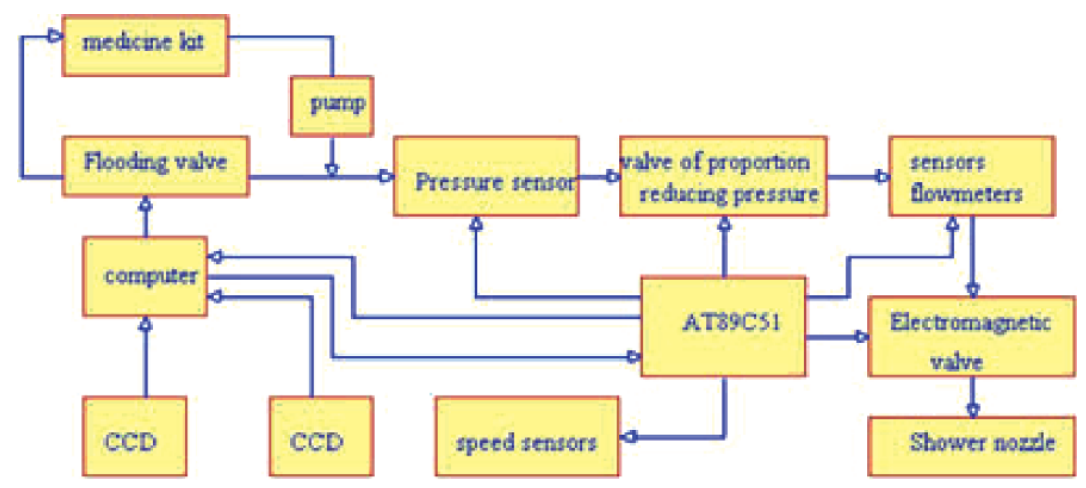

Fig. 1. The total block diagram of the spraying pesticide system

We may know that the spraying pesticide system is composed of medical kit, pump, flow meter, pressure sensor, speed sensors, single-chip, CCD sensor, computer, and shower nozzle from the figure. 


\subsection{Hardware of spraying pesticide control system}

\subsubsection{Components of control system}

The control system is mainly composed of computer, AT89C51 singlechip, interface circuit, photoelectric isolation driving circuit, and A/D converter. The figure 2 is the diagram of the hardware circuit about the control system.

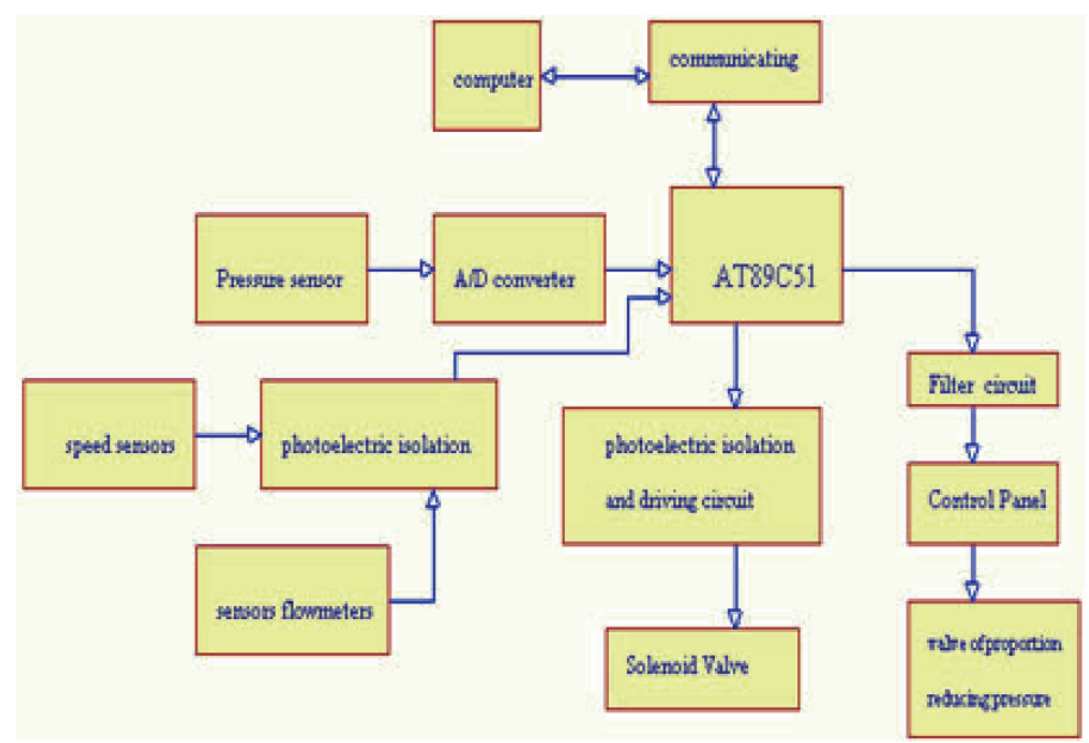

Fig. 2. The diagram of the hardware circuit about the control system

\subsubsection{The control principle}

The single-chip collects pressure signals, flow signals and speed signals in the spraying pesticide system. After preliminary treating, the data is sent to the computer. The computer gives the single-chip an order after it synthetically handles the signal gained and the result of pattern recognition. The single-chip controls valves switch and switch amounts size in control circuit which controls pressure in pipeline, change of pressure controls flow of variable shower nozzles.

\subsubsection{The communicating circuit of the computer and single-chip}

Ways of Computer communication have the parallel communication and the string of lines communication. The speed of the parallel communication 
is much quick, and real time nature is good, but, it is unsuitable to miniaturize a product because it occupies many end lines. Even though the rate of the string of lines communication is much lower, it appears simple, convenient, nimble in the circuit that data handling capacity is not very big (Shi et al. 2002). Therefore, the system adopts the string of lines communication.

Fig. 3 is the figure of the communicating circuit ( $\mathrm{Li}, 2003)$.

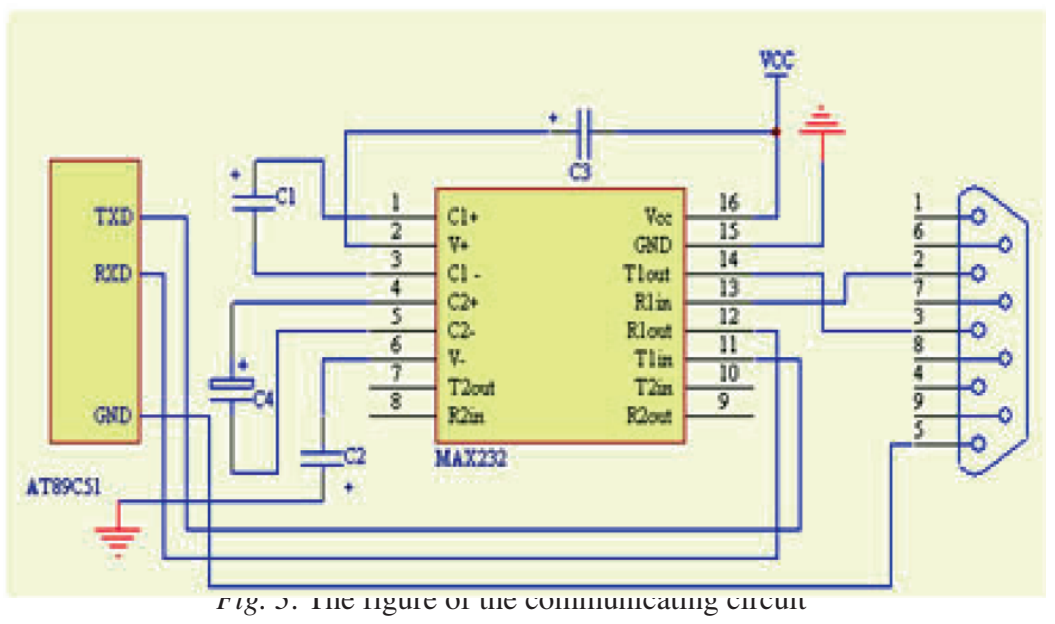

\subsection{The structure of spraying system about fuzzy control}

The mechanical part about the spraying pesticide system exists many uncertain factors in the variable spraying pesticide system. It is difficult to set up the accurate mathematic model, therefore, we applied fuzzy control to the control system. Figure 4 is the structure of spraying system about fuzzy control.

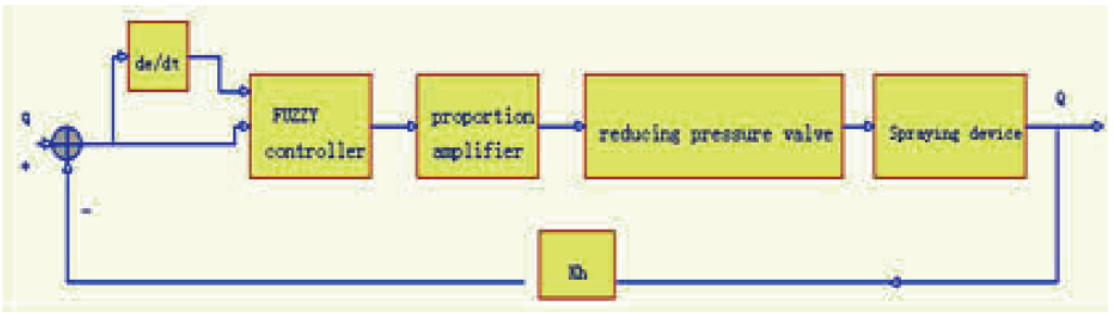

Fig. 4. The structure of spraying system about fuzzy control 


\subsection{The design of fuzzy controller}

\subsubsection{Fuzziness of inputs and outputs}

The fuzzy controller has two inputs and one output. One between of inputs is the difference of flow rate about the input and actual flow rate, another is variable of e: ec=de/dt. The output is u which is the input voltage of the proportion amplifier. E, EC and $\mathrm{U}$ are their fuzzy language variable.

Fuzzy language value:

$\mathrm{E}=\{\mathrm{NB}, \mathrm{NM}, \mathrm{NS}, \mathrm{ZO}, \mathrm{PS}, \mathrm{PM}, \mathrm{PB}\}$

$\mathrm{EC}=\{\mathrm{NB}, \mathrm{NM}, \mathrm{NS}, \mathrm{ZO}, \mathrm{PS}, \mathrm{PM}, \mathrm{PB}\}$

$\mathrm{U}=\{\mathrm{NB}, \mathrm{NM}, \mathrm{NS}, \mathrm{ZO}, \mathrm{PS}, \mathrm{PM}, \mathrm{PB}\}$

\subsubsection{Theory region of fuzzy variables}

The fundamental theory region of $\mathrm{e}$ is $(-\mathrm{e}, \mathrm{e})$. The fundamental theory region of ec is $(-\mathrm{ec}, \mathrm{ec})$. The fundamental theory region of $\mathrm{u}$ is $(-\mathrm{u}, \mathrm{u})$.

Theory region after quantization about e:

$\{-7,-6,-5,-4,-3,-2,-1,0,1,2,3,4,5,6,7\}$

Theory region after quantization about ec:

$\{-6,-5,-4,-3,-2,-1,0,1,2,3,4,5,6\}$

Theory region after quantization about $\mathrm{u}$ :

$\{-6,-5,-4,-3,-2,-1,0,1,2,3,4,5,6\}$

The "a" represents the quantization factor of deviation, "b" represents the quantization factor of deviation change, "c1" represents the proportion factor of control quantity. Therefore,

$$
\begin{aligned}
& a=7 / e \\
& b=6 / e c \\
& c 1=6 / u
\end{aligned}
$$

\subsubsection{Membership functions of fuzzy variables about inputs and outputs}

Fuzzy language value is a fuzzy subset in fact, but language value is described by membership functions ultimately. The membership function of the language value is also known as linguistic semantic rules, it appears with continuous functions sometimes, and it appears with quantification levels of discrete form sometimes. They have their characteristic. For example, the description of continuous functions is accurate; however, the description of 
quantification levels of discrete form is concise, clear and definite. Common membership functions have the triangle function and the Gauss type, membership functions of $\mathrm{E}, \mathrm{EC}$ and $\mathrm{U}$ are all the triangle function in this paper.

\subsubsection{Establishing of fuzzy control rules}

There are no ready-made control regulations in objective world, which are collected by designers who go on observing and extracting experiment data according to the structure of the fuzzy controller, part regulations of the paper are listed.

If $\mathrm{E}$ is $\mathrm{NB}$ and $\mathrm{EC}$ is $\mathrm{NB}$, then $\mathrm{U}$ is $\mathrm{PB}$

If $\mathrm{E}$ is $\mathrm{NM}$ and $\mathrm{EC}$ is $\mathrm{NB}$, then $\mathrm{U}$ is $\mathrm{PM}$

If $\mathrm{E}$ is $\mathrm{NM}$ and $\mathrm{EC}$ is $\mathrm{NM}$, then $\mathrm{U}$ is $\mathrm{PM}$

If $\mathrm{E}$ is $\mathrm{PM}$ and $\mathrm{EC}$ is PS, then $\mathrm{U}$ is $\mathrm{NM}$

\subsubsection{Fuzzy solution}

Purpose of fuzzy solution is to obtain real scatter which may reflect control quantity according to the result of fuzzy reasoning. Common methods have three kinds. The dynamic performance of median method is better than the other; therefore, we collected median method.

\section{RESULTS AND DISCUSSIONS}

MATLAB has a lot of tool case functions to carry on fuzzy analyzing and designing. We can open the artwork interface of Fis editor, conduct fuzziness of inputs and outputs and edit fuzzy rules, when orders about fuzzy are keyed in MATLAB.

Figure 5 is the simulation model of fuzzy control about the spraying pesticide system.

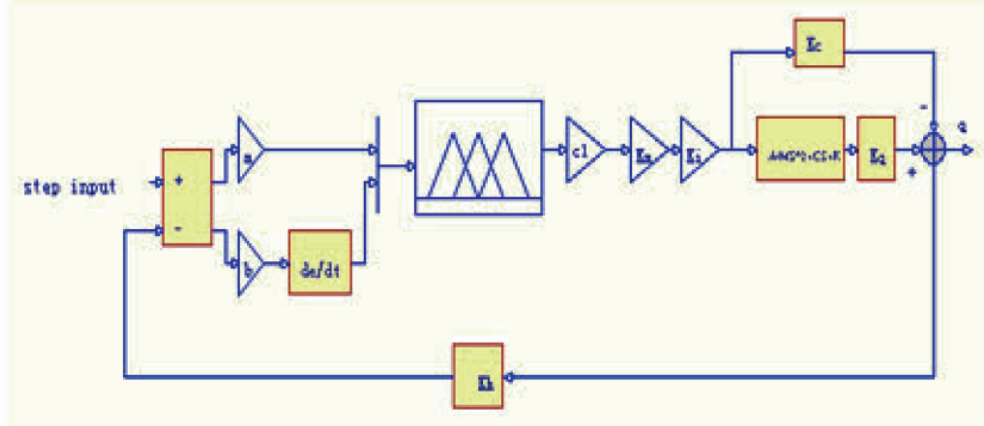

Fig. 5. The simulation model of fuzzy control about the spraying system 
Simulink that MATLAB provides is a software package which can be used to build models, simulate and analyze, and which may realize perfect combination with the fuzzy logic tool case.

After opening the simulation model under the environment of simulink, we strike Fuzzy logic controller, and import the file name of the fuzzy controller which has been designed by us. At last, the ok button is keyed. Fuzzy controller and simulink are linked in this way.

After collecting simulation parameters... under menu simulation, a conversation frame will appear. We set type among solver options to Variable-step, algorithm is order45. Simulation result can be gained from the oscilloscope.

Figure 6 is the result of simulation.

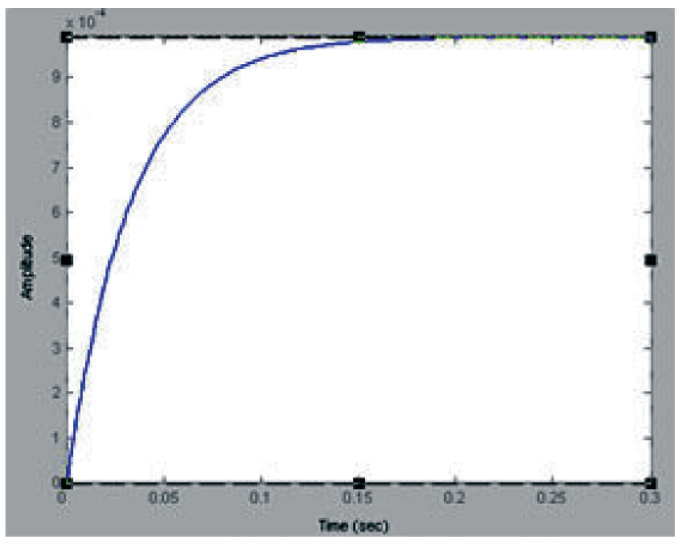

Fig. 6. The result of simulation

We can know transition time is 0.19 second from the figure. Transition time is very short and the system is very stable.

\section{CONCLUSIONS AND FUTURE WORKS}

From the above analysis, the main outcomes can be outlined as follows:

(1) The spraying pesticide control system can satisfy the timely request and the controllability is much better.

(2) Future work is constructing the platform to do dynamic experiment. 


\section{REFERENCES}

Shi Yan 2004. Study on the System of Spraying Rate Varied by Pressure of Liquid Chemical Application, Chinese Agriculture University (in Chinese).

Shi Donghai, Hu Xiao, Zhou Xusheng 2002. Data communicating technology of single-chips from elementary course to mastering, xi' an: Xidian university press, 99 (in Chinese).

Li Chaoqing 2003. Data communicating technology about PC and single-chips with DSP, Beijing: Beihang university press, 30 (in Chinese).

Zhang Guoliang, Zeng Jing, Ke Xizheng, etc. 2002. Fuzzy control and application of matlab, xi'an: xi'an jiaotong university press (in Chinese).

Zhu Jing 1995. Theory of fuzzy control and system principle, Beijing: mechanical industry press (in Chinese).

Zhu Jing 2005. Theory of fuzzy control and system principle, Beijing: mechanical industry press, 233-234 (in Chinese).

Lou Shuntian, Hu Chang-hua, Zhang Wei 2001. System analyzing and designing based on matlab, xi'an: xidian university press (in Chinese).

Sun Liang 2004. matlab language and simulation of control system, Beijing: Beijing industry university press (in Chinese). 